2016 145th TMS Annual Meeting \& Exhibition, Symposium Additive Manufacturing: Building the Pathway towards Process and Material Qualification, February 14 - 18, 2016, Nashville,

Tennessee

\title{
Effect of Printing Orientation on Strength of 3D Printed ABS Plastics
}

\author{
Linlin Cai ${ }^{1}$, Philip Byrd ${ }^{1}$, Hanyin Zhang ${ }^{1}$, Kate Schlarman², Yi Zhang ${ }^{1}$, Michael Golub ${ }^{1}$, Jing \\ Zhang $^{1^{*}}$ \\ ${ }^{1}$ Department of Mechanical Engineering, Indiana University- Purdue University Indianapolis, \\ Indianapolis, IN 46202 \\ ${ }^{2}$ Decatur County Community Schools, Greensburg, IN 47240 \\ *Corresponding author: jz29@iupui.edu
}

Keywords: 3D Printing, Additive Manufacturing, ABS, Mechanical Property

\begin{abstract}
The mechanical strengths of ABS(Acrylonitrile Butadiene Styrene) components fabricated by fused deposition modeling (FDM) technique have been studied, with the focus on the effect of printing orientations on the strength. Using the properties derived from stress-strain curves of the samples, the 0-degree printed sample has the strongest mechanical properties, which is likely due to preferred orientations in individual slice.
\end{abstract}

\section{Introduction}

Fused deposition modeling (FDM) is a type of three-dimensional (3D) printing that constructs physical models from computer aided design (CAD) data. Plastic filament is partially melted then systematically deposited to create one thin layer of the desired object. This process continues, one layer being printed on top of the previous, until the object is complete. The process starts when a 3D model is supplied to the printer's software. The software produces a plan for creating the object by slicing the model into thin horizontal sections. Each section is assigned a tool path that will guide the print head when creating that layer. The tool path consists of a perimeter and a fill. The perimeter, or outline of that slice, is created first. Next the inside is filled with long sweeps, or roads, going back and forth all of which are parallel to each other. The nozzle moves along its tool path and the newly extruded filament bonds with the previously laid down strips. The tool path for each layer is not identical. The parallel long sweeps that make up the fill are rotated slightly with each new layer. Research has been done to study the effects of altering process parameters on the physical properties of the printed object, and process parameters effect on dimensional accuracy and surface finish (Kumar, Ahuja, \& Singh, 2012), and improving the compressive strength (Sood, Ohdar, \& Mahapatra, 2012). In this study, the research is focused on the effect of printing orientation on the tensile strength of 3D printed specimens. 


\section{Methodology}

The specimens used in this study are designed in accordance with the ASTM standard test method for tensile properties of plastics. The printer used is a Dimension SST 3D printer in conjunction with CatalystEx software, both products of Stratasys Inc. The 3D printed specimen material used is ABS, also a product of Stratasys Inc.

The tensile testing is done using a MTS Systems universal testing machine according to ASTM standards for tensile properties of plastics. The model number of extensometer used in the test is 634.12E-54.

Before the tensile tests, the width and thickness of the center section of each of the specimens are measured then entered into the testing program. As shown in Figure 1, the tensile specimen is loaded into the testing machine by attaching the clamps to both ends and the distance between the clamps is measured and entered into the program. Next the extensometer is attached to the center of the specimen. The extensometer will precisely measure the length of that section of the specimen as the tensile testing machine is pulling. The tensile strain rate applied is $0.2 \mathrm{in} / \mathrm{min}(0.0847 \mathrm{~mm} / \mathrm{s})$. The program will use the measurements to calculate strain (relative change in length) and stress (force over area). The program then compiles the raw data from the two components. Exporting the data in a .txt format allows us to view the data in Microsoft Excel.

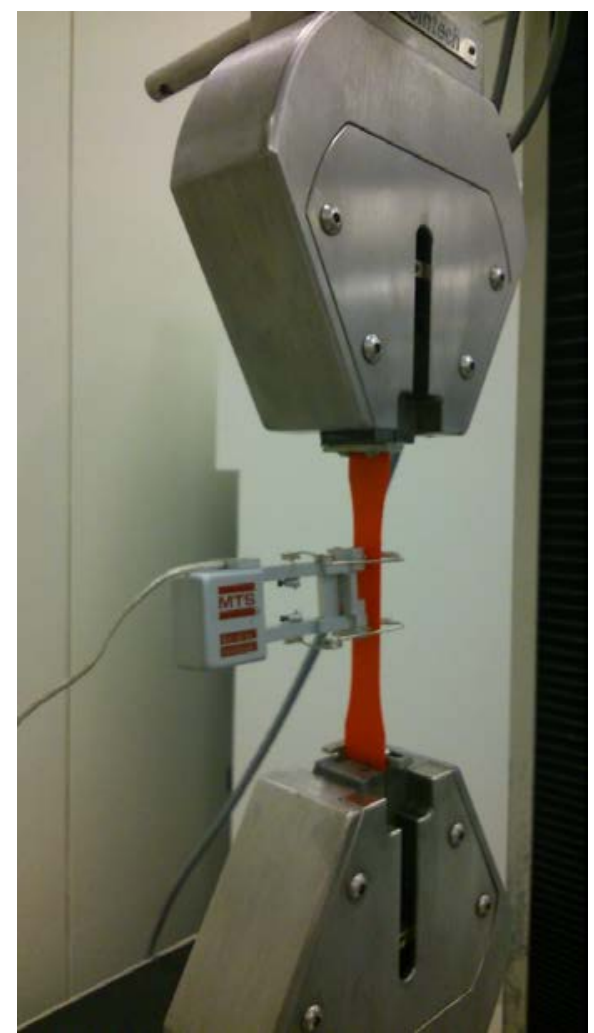

Figure 1: Tensile specimen tested in the MTS testing machine 
Specimens were printed at three orientations: 0 , 45, and $90^{\circ}$ (Figure 2). Printing orientation refers to where in the virtual $\mathrm{x}, \mathrm{y}, \mathrm{z}$ coordinate system of the 3D printer's software the model is placed (Stratasys, 2015a). When an STL file is opened it is placed in a default position.

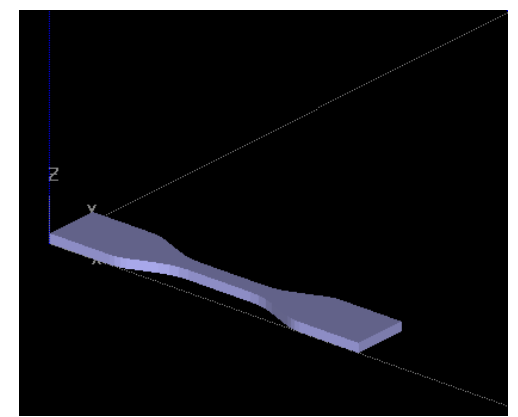

(a)

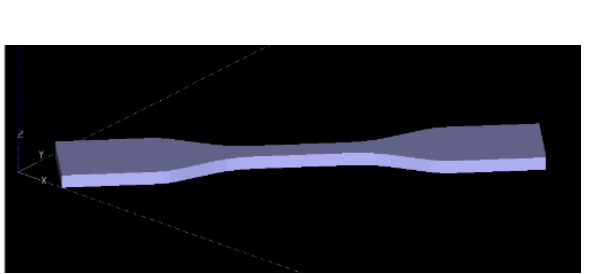

(b)

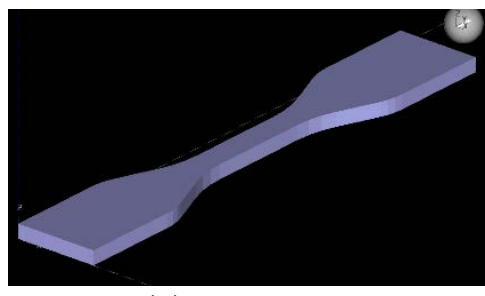

(c)

Figure 2: Printing orientations of the tensile specimens (a) $0^{\circ}$, (b) $45^{\circ}$, and (c) $90^{\circ}$

The 3D printer's software allows manipulation of that position by rotating the model around the $\mathrm{x}, \mathrm{y}$ or $\mathrm{z}$ axis. The specimens were created with their broad side flush with the $\mathrm{x}-\mathrm{y}$ plane at $45^{\circ}$ increments around the $\mathrm{z}$ axis starting with the default position (Figure 3).

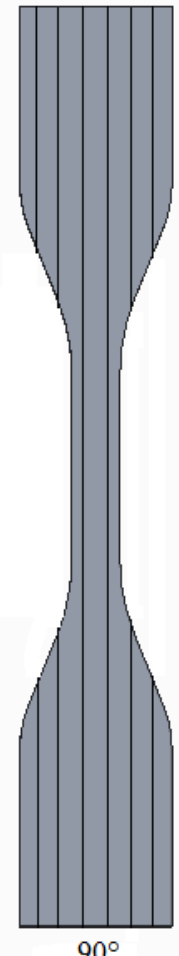

$90^{\circ}$

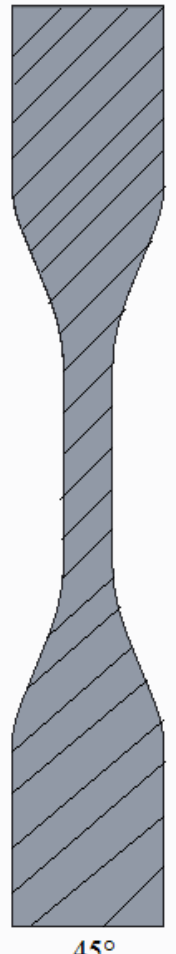

$45^{\circ}$

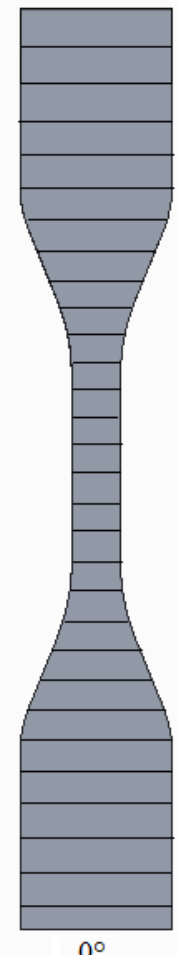

$0^{\circ}$

Figure 3: Illustration of fiber printed orientation in each slice 


\section{Results and discussion}

As an example, figure 4 shows the $0^{\circ}$ tensile bar sample before and after the tensile test. The broken point deviates from the middle of the bar a little bit but they still in the extended part of the bar, which verifies the correctness of the testing method.
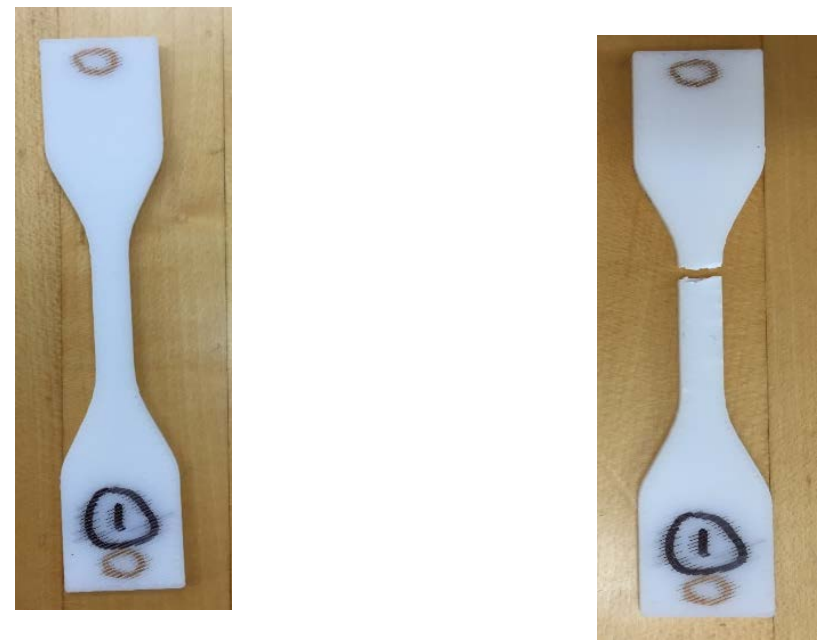

(a)

(b)

Figure 4: Optical images of the $0^{\circ}$ tensile bar (a) before and (b) after the tensile test

The detailed cross sectional view of the fracture surface is given in Figure 5. The smeared surface suggests a ductile fracture. The 10 slices are visible from the left side of the fracture surface. Among these 10 slices, their orientations vary, resulting different facture patterns. The orientation of each slice is increased with a finite angle, so the fibers partially overlap each other in order to achieve maximum contact surface strengths.

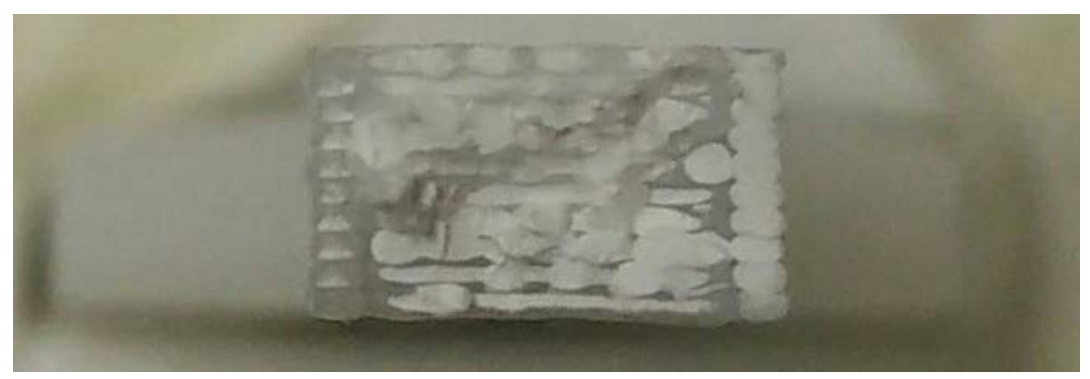

Figure 5: Optical image of cross-section area of the $0^{\circ}$ tensile bar fracture surface

The stress-strain curves of tensile bars in different printing orientations are plotted in Figure 6. It is suggested that the 0 degree orientation, which has the highest peak load and shortest elongation, 
is the strongest one, followed by the 45 degree, and 90 degree orientations respectively. As shown in Table 1, the Young's moduli for the 0 degree, 45 degree, and 90 degree orientations respectively are $1.81 \mathrm{GPa}, 1.80 \mathrm{GPa}$, and $1.78 \mathrm{GPa}$, respectively.

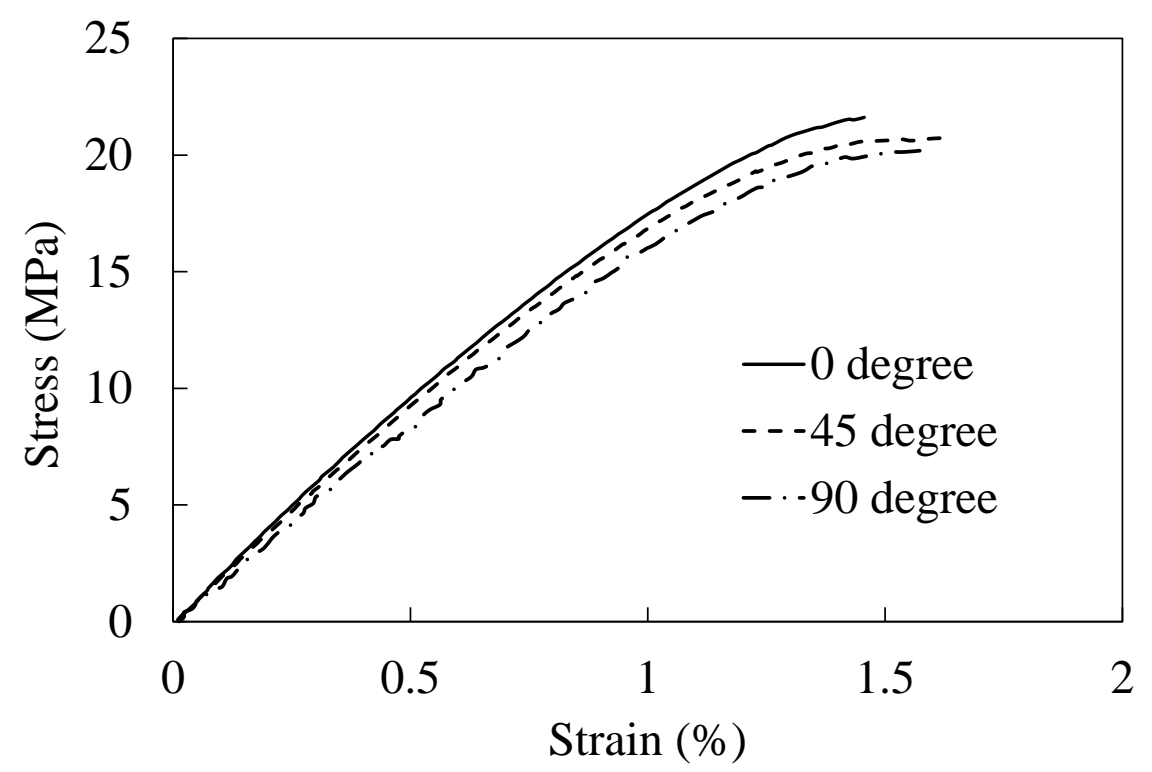

Figure 6. Stress-strain curve of ABS tensile bars in different printing orientations

Table 1: Averaged mechanical strength as a function of printing orientation

\begin{tabular}{|l|l|l|l|}
\hline Printing orientation & $0^{\circ}$ & $45^{\circ}$ & $90^{\circ}$ \\
\hline Young's modulus(GPa) & $1.81+/-0.10$ & $1.80+/-0.11$ & $1.78+/-0.13$ \\
\hline Ultimate strength (MPa) & $22.4+/-0.1$ & $20.7+/-0.1$ & $19.0+/-0.2$ \\
\hline
\end{tabular}

The reason why the $0^{\circ}$ orientation has the highest mechanical properties is that its printing orientation makes the original filament in the individual slice align with the loading force direction. This determines connected point between filaments by fused deposition modeling, to which attention should be paid when designing and application of various printed components.

\section{Summary}

In summary, the 0 degree orientation as it was defined has the highest mechanical properties, as shown with largest Young's modulus and ultimate strength. This result provides information about selecting printing direction during fused deposition modeling for ABS and its characteristic for application. To further understand the mechanics of fused deposition modeling, a detailed analysis of individual slice orientation and their characteristics is needed. 


\section{Acknowledgements}

Linlin Cai and Philip Byrd thank the support provided by the CTEE (Commitment to Engineering Excellence Research Fund) research program and MURI (Multidisciplinary Undergraduate Research Institute) program at Indiana University- Purdue University Indianapolis. Kate Schlarman is supported by NSF (award number: EEC 1406995).

\section{References}

Kumar, P., et al. (2012). "Application of fusion deposition modelling for rapid investment castinga review." International Journal of Materials Engineering Innovation 3(3-4): 204-227.

MTS (2015). "mts.com." from http:/www.mts.com/en/products/producttype/testcomponents/grips-fixtures-accessories/extensometers/index.htm.

MTS (2015). "mts.com." from http://www.mts.com/en/products/producttype/test-systems/loadframes-uniaxial/servohydraulic/standard/index.htm.

Sood, A. K., et al. (2012). "Experimental investigation and empirical modelling of FDM process for compressive strength improvement." Journal of Advanced Research 3(1): 81-90.

Standard, A. (2003). "Standard test method for tensile properties of plastics." ASTM International. Designation: D 638.

Stratasys (2015). CatalystEx, Stratasys.

Stratasys (2015). "stratasys.com." from http://www.stratasys.com/. 